\title{
Validação em Língua Portuguesa da Escala de Motivação de Realização
}

\author{
Thiago Bessa Pontes ${ }^{1}$, Guilhermina Lobato Miranda ${ }^{2}$ \\ thiago.bessa@ufca.edu.br, gmiranda@ie.ulisboa.pt \\ ${ }^{1}$ Instituto de Educação, Universidade de Lisboa, Alameda da Universidade, 1649-013, Lisboa, Portugal \\ ${ }^{2}$ Instituto de Educação, Universidade de Lisboa, Alameda da Universidade, 1649-013, Lisboa, Portugal
}

DOI: 10.17013/risti.27.109-125

\begin{abstract}
Resumo: O objetivo deste trabalho foi validar uma Escala de Motivação de Realização, como primeira aplicação a estudantes portugueses e brasileiros, que aprendem programação informática em $3 \mathrm{D}$. A escala original em língua inglesa foi traduzida para português de Portugal e Brasil, retrovertida para inglês, por falantes das três línguas, até chegar à versão aplicada à nossa amostra, composta por 204 estudantes, 102 brasileiros e 102 portugueses. Determinámos três índices: (i) A Sensibilidade dos itens, (ii) A Validade Fatorial recorrendo à Análise Fatorial Exploratória (AFE) com Rotação Varimax, (iii) e o Alfa de Cronbach. Os resultados podem ser considerados positivos. Os itens revelaram boa ou muito boa sensibilidade; a AFE sugere que a estrutura fatorial proposta pelo modelo teórico não se confirmou na nossa amostra; os Alfas de Cronbach podem ser considerados bons.
\end{abstract}

Palavras-chave: Análise Fatorial; Escala de Motivação de Realização; Programação Computacional; Python para CAD; Validação de uma Escala

\section{Validation in Portuguese Language of the Achievement Motivation Inventory}

\begin{abstract}
This research aims to validate the Achievement Motivation Inventory with a sample of Portuguese and Brazilian students who learn programming computer using $3 \mathrm{D}$ tools. The original Scale is in English was translated to Portugal and Brazilian Portuguese, and after retranslated to English, by three languages speakers. The final version was applied to our sample: 204 students, 102 Brazilians and 102 Portuguese. To the validation process, we determined three indexes: (i) the Sensibility of each item of the Scale, (ii) the Factorial Validity using Exploratory Factor Analysis (EFA) with Varimax Rotation, (iii) and the Cronbach Alpha. The results can be considered positive. The items presented a good or very good Sensibility; the EFA suggests that the factor structure proposed by the theoretical model was not confirmed in our sample; Cronbach's Alphas can be considered good.
\end{abstract}

Keywords: Achievement Motivation Inventory; Computer Programming; Factor Analysis; Inventory Validation; Python to CAD. 


\section{Introdução}

A "programação de computadores" nos primeiros anos do ensino superior, para os alunos que escolheram a computação e cursos afins, é um desafio para os professores e levanta dificuldades de aprendizagem, assunto largamente analisado na literatura especializada (Dan, Cooper \& Paush, 2000; Jenkins, 2002; Moons \& Backer, 2013; Pea \& Kurland, 1984; entre outros). Ensinar programação a alunos fora das áreas da computação e das tecnologias digitais é um desafio ainda maior, pois não é clara para estes a necessidade de aprender a programar, e faltam-lhes as bases matemáticas para concretizar uma programação de qualidade.

A literatura especializada no domínio da aprendizagem inicial da programação de computadores para os estudantes de informática, tem analisado as dificuldades que muitos encontram, entre elas: o raciocínio abstrato, as heurísticas de resolução de problemas, os erros de sintaxe e a algoritmia. Contudo, estudos como os de Caspersen e Kölling (2009) apontam que a maior dificuldade dos iniciantes está em combinar a utilização dos conceitos básicos de programação e o seu uso efetivo na codificação. Os alunos entendem os conceitos das estruturas das linguagens de programação, mas não sabem como utilizá-los no código.

As dificuldades que os estudantes encontram no seu percurso de aprendizagem podem influenciar a motivação e levar a que desejem abandonar os estudos neste domínio. Para Lemos (2015) a motivação deve ser valorizada nos contextos escolares pois produz uma melhor aprendizagem e tem influência no desempenho, na confiança em si próprio e produz uma maior satisfação na realização dos trabalhos.

A motivação aqui estudada é a Motivação de Realização baseada na Teoria de Atkinson. Para se alcançar o sucesso na realização de uma dada tarefa, o aluno deverá manifestar interesse e empenho, tendo em vista obter sucesso ou, pelo contrário, evitar o fracasso, que geralmente se expressa pela ansiedade e inibição, tendo consequências negativos no comportamento (Jesus, 2000).

A utilização de provas para avaliar construtos psicológicos, como é o caso da motivação de realização, pressupõe que os instrumentos que vamos usar devem ser tratados com rigor e cientificidade. Negligenciar as ações de validação dessas provas pode por em causa a credibilidade da investigação, pois esta depende do controlo das variáveis que podem ameaçar a validade interna e externa dos planos de investigação (cf. Almeida \& Freire, 2017; Cohen, Manion \& Morrison, 2006; Tuckman, 2014; entre outros), onde o uso de instrumentos credíveis é essencial.

A escala aqui estudada foi desenvolvida em língua inglesa por Muthee e Thomas, (2011), com base na teoria de Atinkson sobre a Motivação de Realização, e aplicada a uma amostra da população estudantil do ensino secundário de Nairóbi. Esta escala possui 32 itens, 18 estão redigidos de forma positiva e 14 de forma negativa. Os itens com formulação positiva e negativa estão dispostos aleatoriamente na escala.

Após a autorização dada pelos autores, foi feita a tradução e retroversão, mantendo o enunciado de cada questão o mais próximo possível da versão original (Runa \& Miranda, 2015). A segunda etapa consistiu em testar esta primeira versão junto de 11 alunos, que foram entrevistados após a aplicação da escala. Realizou-se uma análise de conteúdo 
no qual foram feitas melhorias no que diz respeito à tradução para a língua portuguesa (versão de português de Portugal e versão de português do Brasil). Na terceira etapa foi aplicada a escala a uma amostra portuguesa e brasileira $(n=204)$ utilizando três índices para assegurar a credibilidade do instrumento: a sensibilidade dos itens, a validade fatorial e a fiabilidade pelo alfa de Cronbach.

Com os resultados alcançados concluímos que o instrumento de medida é credível para mensurar a motivação de realização em amostras retiradas de populações que falam a língua portuguesa. Entende-se que um trabalho continuado de validação em diferentes amostras é necessário para confirmar estes resultados.

\section{Fundamentação Teórica}

Ao realizar a revisão de literatura verificámos que a motivação é considerada como um impulsionador da ação, que pode determinar comportamentos específicos (cf. Chiavenato, 1999, Lemos, 2015, entre outros). Os autores Taipa e Fita (2015) consideram que "a motivação é um conjunto de variáveis que ativam a conduta e a orientam em determinado sentido para poder alcançar um objetivo” (p. 77)

A motivação escolar é uma categoria da motivação e tem tanta importância como a motivação para realizar outras atividades. Para Lemos (2015) os alunos motivados são os que farão um percurso escolar mais longo, pois eles otimizam a aprendizagem e o desempenho e manifestam mais entusiasmo, curiosidade e interesse.

Existem dois tipos fundamentais de objetivos que determinam a motivação escolar: objetivos de aprendizagem e objetivos de realização ou desempenho (Elliot \& Dweck, 2005). Os primeiros induzem os estudantes a serem persistentes, a atribuírem os seus sucessos e fracassos ao esforço e a causas controláveis, porque estão mais interessados em desenvolver as competências associadas à aprendizagem das diferentes tarefas do que ao reforço do ego. Pelo contrário, os estudantes que se motivam para atingir objetivos de realização têm tendência a procurar atividades em que possam obter juízos favoráveis sobre os seus desempenhos pois, caso contrário, podem ficar fragilizados enquanto indivíduos (Dweck \& Leggett, 1988). Segundo Lieury e Fenouillet (1997) esta distinção entre objetivos de realização e de aprendizagem "é semelhante à distinção entre um envolvimento em relação ao ego (objectivo de desempenho) e um envolvimento em relação à tarefa (objectivo de aprendizagem), que é retomada por muitos autores (p. 81)”.

Ramos (2013) afirma que, no contexto académico do ensino superior, a motivação é decisiva para a qualidade da aprendizagem e do desempenho. Ela alega que os alunos que estão motivados apresentam um comportamento ativo no processo de aprendizagem, o que o possibilita melhor absorção do domínio do conteúdo estudado, mas claro, com o dispêndio de esforço e dedicação adequado.

Compreender como os fatores motivacionais podem influenciar a aprendizagem da programação junto de estudantes de arquitetura, cujas motivações académicas e profissionais se situam noutros domínios do conhecimento, pode ser desafiador pois, não sendo em princípio a programação um assunto que lhes interesse, terão que a aprender para finalizar os seus estudos universitários e para responderem às demandas do mundo profissional. 
Preparar profissionais para o futuro é o papel de qualquer professor, e o uso das tecnologias pode ser motivador para esse percurso. Desta forma, Beirão afirma que: "É neste campo que a formação do arquitecto se encontra muito aquém do que deveria ser a sua formação ideal. Argumento que a sua educação avançada em novas tecnologias, nas áreas de multimédia, computação e ferramentas digitais em geral, constituem uma mais-valia muito especial" (2017, Secção As valências da formação em arquietctura, par. 5)

Celani (2008) reforça esta ideia quando diz que: "a programação pode melhorar o raciocínio lógico e o pensamento conceitual no design. Minhas conclusões são tiradas sobre o desenvolvimento histórico do software $\mathrm{CAD}$, sobre experiências pedagógicas com crianças e estudantes de arquitetura e, finalmente, sobre algumas aplicações recentes de programação em projetos arquitetônicos”. (p. 2)

Para a programação de projetos arquitetônicos, o uso dos conceitos de programação de computadores para a modelagem paramétrica, melhora e auxilia o arquitecto facilitando o seu trabalho, fazendo com que ele possa criar modelos complexos mudando algumas variáveis numéricas, em curto período e com alta eficiência, como relatam os estudos de Santos e Beirão (2017).

Fonseca, Pifarré e Redondo (2013) confirmam ao dizer: “centrados en el ámbito arquitectónico, las formas de expresión y comunicación tradicionales como el panel impreso o la maqueta física se están viendo complementadas e incluso sustituidas por el uso de todo tipo de herramientas TIC: desde las avanzadas simulaciones virtuales o la visualización mediante realidad aumentada de modelos superpuestos con la información tanto real como virtual, hasta los ya incluso clásicos montajes fotográficos en paneles compositivos, la visualización multi-formato de ficheros CAD (Computer Assisted Design) y más recientemente su evolución en los formatos BIM (Building Information Modeling)” (p. 2)

O problema que se nos colocou foi que instrumento usar para medir a motivação dos estudantes que participaram na investigação. Tendo em conta o que antes referimos sobre os objetivos que geram a motivação para as tarefas escolares, optámos por uma escala que mede a Motivação de Realização, fundamentada na teoria desenvolvida por Atkinson, McClelland, Clarck e Lowell (1953), pois esta adequava-se ao ambiente de estudo que investigámos.

\subsection{Motivação de Realização}

Muthee e Thomas (2011) definem a motivação de realização como um conceito amplo. Uma variável de personalidade que tem sido usada para explicar as diferenças individuais em vários contextos, incluindo a escola, o desporto e o trabalho. Trata-se de um conceito multidimensional que necessita de clarificação e de definições operacionais que permitam desenvolver um instrumento de medição.

A Teoria da Motivação de Realização teve como percursor, segundo Covington (1998), o estudo de Ferdinand Hoppe, realizado nos anos 30, como "a chave para a questão de como, psicologicamente, os seres humanos definem sucesso e fracasso” (p. 27). Hoppe convidou estudantes universitários e pessoas do comércio local para participar do experimento, que consistia em lançar anéis em estacas móveis a diferentes distâncias. Ele descobriu que alguns sujeitos se sentiam satisfeitos depois de acertar em torno 
de oito anéis, enquanto outros apresentaram frustração mesmo após terem feito doze lances assertivos. Hoppe concluiu que o nível de desempenho necessário para despertar sentimentos de sucesso, muda ao longo do tempo e de indivíduo para indivíduo (Covington, 1998).

O estudo de Hoppe levou Covington (1998) a concluir que há vários fatores de motivação como: Níveis de Aspiração, Autoconfiança, Expectativa, Desafios Realistas, Metas Autogeradas e Controlo do Progresso Próprio. (pp. 28-32).

Hoppe inspirou o artigo The Achievement Motive (1953) de Atkinson, Clark e Lowell e influenciou McClelland no seu programa de investigação sobre motivação na Universidade de Michigan.

Atkinson (1957) relata que "a motivação de realização é um modelo teórico destinado a explicar como o motivo para obter sucesso e o motivo para evitar o fracasso influenciam o comportamento numa situação em que o desempenho é avaliado em relação a algum padrão de excelência” (p. 371).

Na teoria de Atkinson temos a presença da atividade orientada para a realização, definida como uma atividade em que há no sujeito uma expectativa de que o seu desempenho será aferido a partir de um padrão que deseja alcançar a excelência. Desta forma o sujeito é desafiado a realizar ações que o conduzam ao sucesso esperado, porém há também ameaças de um possível fracasso. As atividades orientadas para a realização são influenciadas por duas intenções antagônicas que são: as intenções para alcançar o sucesso e as intenções para evitar o fracasso.

Os estudos de Atkinson e Feather (1969) referem que a teoria da motivação de realização se concentra principalmente na resolução do conflito entre estas duas intenções opostas que são inerentes a qualquer atividade orientada para a realização, mas também ressaltam que há fontes extrínsecas de motivação para realizar uma atividade que influenciam diretamente os resultados motivacionais (p. 338). Contudo, cada pessoa desenvolve, ao longo do seu percurso existencial, um padrão motivacional que pode ser mais orientado para obter o sucesso ou, pelo contrário, para evitar o fracasso. São dois padrões distintos que se repercutem no modo como cada um enfrenta as diferentes tarefas escolares e profissionais. Geralmente, os alunos motivados para obter sucesso são mais persistentes, mesmo quando encontram obstáculos para alcançar os objetivos propostos, resistem melhor à frustração e avaliam melhor as situações. Os estudantes com um padrão motivacional 'para evitar o fracasso', resistem pior à frustração decorrente dos obstáculos que encontram no processo de aprendizagem, são menos persistentes e tem tendência a escolher tarefas ou muito fáceis ou muito exigentes, pois avaliam pior as situações que lhe são propostas (cf. Deci \& Ryan, 1985; Vallerand, 1993; citados por Lieury \& Fenouillet, 1997).

\subsection{Escala de Motivação de Realização}

A Escala de Motivação validada neste estudo baseia-se na conceção de motivação de realização que acabámos de descrever. Conforme foi referido na Introdução foi desenvolvida por Muthee e Thomas (2011), para determinar os padrões motivacionais de estudantes do ensino secundário de escolas da cidade de Nairobi, tendo como objetivo o desenvolvimento de estratégias de intervenção eficazes para melhorar o desempenho dos alunos. Visou ainda sugerir mudanças nas políticas governamentais de educação, tendo 
em vista promover padrões motivacionais nos estudantes mais adequados a melhorar os seus desempenhos. O estudo original analisou aspetos que podem influenciar o desempenho como: o ambiente familiar, o estatuto socioeconómico da família, o clima da sala de aula, as habilidades cognitivas e a motivação de realização.

A Escala de Motivação de Realização foi originalmente desenvolvida a partir das seguintes etapas:

a. Conceptualização:

Foi feita uma revisão da literatura teórica e empírica, dos testes disponíveis e consulta a psicólogos sobre motivação de realização; foram identificadas quatro dimensões:

- I - Motivação de Realização (Motivation for Achievement): caracterizada pela competitividade e orientação para alcançar objetivos.

- II - Recursos Internos (Inner Resources): caracterizada pelo estilo descontraído, felicidade, paciência e autoconfiança.

- III - Forças Pessoais Internas (Inter Personal Strengths): caracterizada pela assertividade, diplomacia pessoal, extraversão e co-cooperatividade.

- IV - Hábitos de Trabalhos (Work Habits): caracterizada pelo planeamento e organização, iniciativas e espírito de equipa.

b. Geração e seleção dos itens:

Os itens foram desenvolvidos em forma de proposições, tendo o cuidado de incluir frases formuladas pela positiva e frases formuladas pela negativa. Para garantir a validade do conteúdo da escala, foi preparado um grande número de itens tendo como base as dimensões antes referidas, que resultou num número de 80 itens. Após uma análise minuciosa, foram retirados itens repetitivos, sobrepostos e ambíguos, resultando uma lista de 50 itens. Essa nova lista encurtada passou por uma nova filtragem, resultando numa terceira versão com 40 itens, sendo 24 formulados pela positiva e 16 itens pela negativa. Para a inclusão dos melhores itens foi realizado um teste $t$-student a partir do método de Likert (Edwards, 1957), de modo a selecionar os 25\% superiores e inferiores da amostra. Os itens que produziram valores $t$ estatisticamente significativos foram os primeiros 32 itens, variando entre 6,955 a 2,615, que produziram a escala final. Os demais oito foram retirados da escala. Entre os 32 itens retidos na escala final, 18 foram formulados pela positiva e 14 pela negativa. Os itens foram dispostos aleatoriamente na escala final. Os itens com formulação positiva são: $3,4,5,6,11,13,14,16,17,20,23,24,26,28,29,30,31$; e com formulação negativa são: 1, 2, 7, 8, 9, 10, 12, 15, 18, 19, 21, 22, 25 e 27.

c. Pontuação:

As respostas aos itens foram registadas numa escala de cinco pontos de Likert, onde 5 representa que o respondente está "completamente de acordo" com a afirmação, 4" quase sempre de acordo", 3 "parcialmente de acordo", 2" quase sempre em desacordo" e 1 "completamente em desacordo". Aquando da inserção dos itens na base de dados para realizar o tratamento estatístico de validação, nos itens formulados pela positiva mantivemos os valores da escala e nos itens 
formulados pela negativa tivemos a necessidade de inverter os valores (Maroco, 2014). As pontuações mais altas indicam níveis mais elevados de motivação de realização, e as pontuações mais baixas níveis mais baixos.

\section{d. Confiabilidade}

Na escala original apenas foi determinado o alfa de Cronbach, tendo em vista garantir a confiabilidade da escala. Obteve um valor de 0.749 , que pode ser considerada uma boa consistência interna (Maroco \& Garcia-Marques, 2013).

\section{Método}

\subsection{Participantes}

Foi constituída uma amostra não probabilística de alunos utilizadores de ferramentas de desenho assistido por computador (CAD), de Portugal e Brasil. Em Portugal, na cidade de Lisboa, foram coletados 109 inquéritos em papel, em cinco turmas diferentes: a primeira, composta por 30 estudantes no Instituto Superior Técnico da Universidade Lisboa, nas aulas de Programação e Computação para Arquitetura; a segunda, terceira e quarta turmas, composta respetivamente por 18, 29 e 17 estudantes da disciplina de Modelação e Visualização Tridimensional da Faculdade de Arquitetura da Universidade de Lisboa (FAUL); e a quinta turma composta por 15 estudantes da disciplina de Projeto Urbano Paramétrico também da FAUL. Dos 109 inquéritos coletados 7 continham inconsistências nas respostas e foram descartados, totalizando 102 inquéritos válidos coletados em Portugal.

No Brasil foram coletados 102 inquéritos em formato digital, junto de estudantes do Curso de Arquitetura da Faculdade Paraíso, da Universidade Federal do Ceará, da Universidade de Fortaleza, e da Faculdade de Juazeiro do Norte, com o total de 102 questionários. Por fim resultou numa amostra total de $204(n=204)$.

A amostra é composta por $52 \%$ estudantes do género feminino e $48 \%$ do masculino, com idades compreendidas maioritariamente entre os 20 e 30 anos (74\%), solteiros em 95\% e casados nos $5 \%$ restantes.

\subsection{Procedimentos}

Após selecionada a escala que nos pareceu mais adequada para atingir o objetivo do estudo, procedeu-se ao processo de validação para a nossa amostra. O primeiro passo foi traduzir da língua inglesa para a língua portuguesa (versão português de Portugal e português do Brasil) e retroverter para inglês, tentando manter a maior fidedignidade ao sentido original dos itens (Moreira, 2004).

Depois, o questionário foi aplicado a uma amostra de 11 alunos e foram feitas entrevistas registradas em áudio. Realizou-se uma análise de conteúdo, na qual foram detetadas sete sugestões de melhorias, tendo sido aceites duas, que deram origem a pequenos ajustes no texto para expressões em português de Portugal e em português do Brasil.

A versão final da escala em língua portuguesa foi aplicada à nossa amostra $(n=204)$, sendo que a versão portuguesa foi aplicada à amostra de estudantes portugueses e a versão brasileira à amostra de estudantes brasileira $(\mathrm{n}=102)$. 
Usámos o SPSS (versão 24) para tratamento e análise dos dados.

\section{Resultados}

\subsection{Sensibilidade dos Itens, Validade Fatorial e Consistência Interna}

\subsubsection{Sensibilidade}

A análise da sensibilidade dos itens permite verificar se a escala é sensível às respostas diferenciadas dos sujeitos que são estruturalmente diferentes no constructo que se está a medir. Dito de outro modo, verifica se os itens da escala permitem discriminar os sujeitos com padrões motivacionais diferentes. É realizada recorrendo a dois indicadores: a assimetria (skewness) e o achatamento (kurtosis) que, segundo Maroco (2014), tem valores aceitáveis quando variam entre -3 e +3 para o skewness e -7 e +7 para o kurtosis. De acordo com nossa análise, os 32 itens que compõem a escala têm valores de assimetria e de achatamento aceitáveis, conforme se pode observar na tabela 1. Por isso, nesta primeira fase de análise resolvemos não eliminar nenhum item.

\begin{tabular}{|c|c|c|}
\hline Variável & Skewness & Kurtosis \\
\hline V1. Sinto que sou uma pessoa preguiçosa & -0.207 & -0.682 \\
\hline V2. Muitas vezes passam-se dias sem eu ter feito nada. & -0.486 & -0.968 \\
\hline $\begin{array}{l}\text { V3. Gosto de ler a biografia de grandes pessoas, a fim de aprender como elas } \\
\text { superaram obstáculos e alcançaram grandes coisas na vida. }\end{array}$ & 0.182 & -0.781 \\
\hline $\begin{array}{l}\text { V4. Planeio com antecedência quais os assuntos a estudar durante o meu } \\
\text { tempo livre. }\end{array}$ & -0.316 & -0.627 \\
\hline $\begin{array}{l}\text { V5. Quando fico a saber que alguém que gosta de mim conseguiu alcançar algo } \\
\text { grandioso, fico motivado para fazer a mesma coisa de uma maneira melhor. }\end{array}$ & -0.932 & 0.412 \\
\hline $\begin{array}{l}\text { V6. A maioria das pessoas que me conhecem dizem que eu sou trabalhador e } \\
\text { ambicioso. }\end{array}$ & -0.23 & -0.733 \\
\hline V7. Vou adiando o que eu deveria estudar no dia-a-dia. & 0.027 & -1.008 \\
\hline V8. Levo muito tempo para começar a estudar. & -0.033 & -1.072 \\
\hline $\begin{array}{l}\text { V9. Na maioria dos dias prefiro descontrair e relaxar em vez de me preparar } \\
\text { para o próximo dia de trabalho na Faculdade. }\end{array}$ & -0.006 & -0.878 \\
\hline V10. Às vezes esqueço-me de fazer os trabalhos de casa. & -0.423 & -0.903 \\
\hline V11. Nunca deixo uma tarefa inacabada, pois termino tudo o que começo. & -0.193 & -0.87 \\
\hline $\begin{array}{l}\text { V12. Gosto de trabalhar com pessoas que tenham resultados do meu nível ou } \\
\text { inferior, mais do que com aquelas que são mais inteligentes e trabalhadoras do } \\
\text { que eu. }\end{array}$ & -1.09 & 0.279 \\
\hline V13. Não gosto de falhar nos exames devido a não me ter preparado. & -1.192 & 0.591 \\
\hline $\begin{array}{l}\text { V14. Trabalho sempre muito para estar entre os melhores alunos da minha } \\
\text { Faculdade. }\end{array}$ & -0.017 & -0.665 \\
\hline V15. Sinto que vivo a vida como ela é, sem a planear. & -0.097 & -1.005 \\
\hline V16. Tenho como objetivo alcançar o mais alto nível na Educação. & -0.681 & -0.041 \\
\hline
\end{tabular}




\begin{tabular}{|c|c|c|}
\hline Variável & Skewness & Kurtosis \\
\hline V17. Quando crescer, quero fazer algo que os outros não fizeram. & -0.842 & 0.138 \\
\hline $\begin{array}{l}\text { V18. Sou basicamente uma pessoa competitiva e compito apenas por uma } \\
\text { questão de competir. }\end{array}$ & -0.178 & -0.723 \\
\hline $\begin{array}{l}\text { V19. Acredito que o sucesso na vida tem menos a ver com o trabalhar no duro, } \\
\text { e mais a ver com a sorte e com estar no lugar certo na hora certa. }\end{array}$ & -0.348 & -0.827 \\
\hline $\begin{array}{l}\text { V2o. Gosto de ler todos os tipos de livros, incluindo aqueles que não fazem } \\
\text { parte dos programas académicos. }\end{array}$ & 0.017 & -1.126 \\
\hline $\begin{array}{l}\text { V21. Ficarei satisfeito com um desempenho acima da média, mesmo que não } \\
\text { seja o meu melhor. }\end{array}$ & 0.452 & -0.458 \\
\hline $\begin{array}{l}\text { V22. Prefiro usar o meu tempo para fazer qualquer outra coisa, ao contrário de } \\
\text { tentar aperfeiçoar algo que já tenha concluído. }\end{array}$ & -0.14 & -0.84 \\
\hline $\begin{array}{l}\text { V23. Gosto de passar a maior parte do meu tempo sozinho, concentrado no } \\
\text { meu trabalho académico. }\end{array}$ & 0.287 & -0.477 \\
\hline $\begin{array}{l}\text { V24. Tento sempre destacar-me do resto da minha turma, de uma forma ou de } \\
\text { outra. }\end{array}$ & 0.097 & -0.641 \\
\hline $\begin{array}{l}\text { V25. Só levo a cabo os meus planos se estiver certo de que outras pessoas os } \\
\text { vão aprovar. }\end{array}$ & -0.36 & -0.532 \\
\hline V26. Fico inquieto e irritado quando sinto que estou perdendo tempo. & -1.181 & 0.95 \\
\hline $\begin{array}{l}\text { V27. Não é uma boa ideia ter sempre melhores resultados do que os outros, } \\
\text { porque isso pode fazê-los sentirem-se mal consigo próprios. }\end{array}$ & -0.831 & -0.346 \\
\hline V28. Gosto de ser o melhor aluno da minha turma. & -0.142 & -0.547 \\
\hline $\begin{array}{l}\text { V29. Gosto de terminar as minhas tarefas académicas, mesmo quando são } \\
\text { dificeis e consomem muito tempo. }\end{array}$ & -1.21 & 1.073 \\
\hline $\begin{array}{l}\text { V3o. Gosto de fazer amizade com o aluno mais inteligente da minha turma, de } \\
\text { modo a manter os meus padrões de desempenho. }\end{array}$ & 0.007 & -0.942 \\
\hline $\begin{array}{l}\text { V31. Gosto quando as pessoas dizem na frente dos outros que estou indo bem } \\
\text { na Faculdade. }\end{array}$ & -0.468 & -0.607 \\
\hline $\begin{array}{l}\text { V32. Gostaria de lidar com situações difíceis, de modo que a culpa ou o louvor } \\
\text { pelos resultados obtidos, fossem apenas a mim dirigidos. }\end{array}$ & -0.133 & -0.951 \\
\hline
\end{tabular}

Tabela 1 - Índice de sensibilidade da escala de motivação de realização: Assimetria (skewness) e Achatamento (kurtosis)

\subsubsection{Análise Fatorial}

O indicador mais importante, quando se pretende determinar a credibilidade científica de um instrumento de medida que avalia um constructo psicológico, é a validade. Há vários tipos de validade (Anastasi \& Urbina, 1997; Moreira, 2004), onde destacamos a validade de conteúdo e a validade de constructo. A validade de conteúdo foi determinada pelo estudo original, e a de constructo deve ser sempre verificada em cada novo estudo que se realiza com amostras diferentes, para verificar se o instrumento se comporta de maneira idêntica. No estudo original, os autores (Muthee \& Thomas, 2011) não realizaram ou, pelo menos, não divulgaram a análise fatorial. No entanto, pareceu-nos essencial determinar este índice de validade do constructo que se está a medir para verificar se, na amostra a que se aplica a escala, as respostas se organizam de acordo com o constructo teórico proposto. A teoria prevê, 
como antes referimos, que o Constructo de Motivação de Realização que é avaliado por esta Escala é multidimensional, i.e., é composto por mais do que uma dimensão, neste caso por quatro dimensões: (i) Motivação de Realização, (ii) Recursos Internos; (iii) Forças Pessoais Internas; (iv) e Hábitos de Trabalho. No estudo original os autores não discriminam os itens que fazem parte de cada dimensão. Por isso, resolvemos fazer uma análise ao conteúdo de cada item e integrar os diferentes itens em cada uma das quatro dimensões. O resultado desta análise pode ser observado na Tabela 2, que cumpre com o critério assinalado por Maroco (2014), que refere que cada dimensão deve incluir no mínimo 3 itens.

\begin{tabular}{llll}
\hline Dimensão 1 & Dimensão 2 & Dimensão 3 & Dimensão 4 \\
\hline$V_{3}, V_{13}, V_{14}, V_{16}, V_{17}$, & $V_{1}, V_{2}, V 7, V 8, V 9, V 10$, & $V_{12}, V_{19}, V_{25}, V_{27}, V_{30}$, & $V_{4}, V_{5}, V 6, V 11, V 20$, \\
$V_{18}, V_{24}, V 26, V 28$ & $V_{15}, V_{21}, V_{22}$ & $V_{31}, V_{32}$ & $V_{23}, V_{29}$ \\
\hline
\end{tabular}

Tabela 2 - Relação dos itens por dimensão

Seguidamente realizámos uma Análise Fatorial Exploratória (AFE), com Rotação Varimax (Kaiser, 1958) com os 32 itens da escala, para verificar as comunalidades dos itens e se se organizavam nos quatro fatores/dimensões de acordo com a teoria.

A AFE deve obedecer a determinados pressupostos, quer dizer, só é aconselhável avançar com esta análise se o valor de KMO (Kaiser-Meyer-Olkin Measure) for igual ou superiores a .70 e se o valor do Teste de Esfericidade de Bartlett (Bartlett's Test of Sphericity) for igual ou inferior a .001. No nosso caso estes valores foram cumpridos, conforme se pode ler na Tabela 3 .

KMO and Bartlett's Rest

\begin{tabular}{lll}
\hline Kaiser-Meyer-Olkin Measure of Sampling Adequacy. & \multicolumn{2}{l}{.767} \\
\hline \multirow{3}{*}{ Bartlett's Test of Sphericity } & Approx. Chi-Square & 1877.637 \\
\cline { 2 - 3 } & $\mathrm{df}$ & 496 \\
\cline { 2 - 3 } & Sig. & .000 \\
\hline
\end{tabular}

Tabela 3 - Teste de KMO e Bartlett's com 32 itens

Os resultados desta primeira AFE mostraram o contributo de cada item para a variância total explicada; os 10 fatores que emergiram da análise representavam $63 \%$ da variância total da escala. Resolvemos não aceitar esta primeira análise pois havia uma grande dispersão dos 32 itens por 10 fatores, longe da proposta teórica original. Resolvemos forçar esta análise a n fatores até aos 4 propostos pela teoria, mas com uma variância total explicada de apenas de 40\%, o que não nos pareceu aceitável (cf. Moreira, 2004). Por isso tentamos novas abordagens tendo chegado a uma escala com 21 itens dos 32 iniciais, que se organizaram em 5 fatores, que explicam quase $56 \%$ da variância total $(55,688 \%)$, o que já nos pareceu aceitável para uma primeira AFE, conforme se pode observar na tabela 4 (pressupostos da AFE) e na tabela 5 (AFE com Rotação Varimax aos 21 itens). Esta escala de 21 itens foi obtida eliminando os que saturavam em dois fatores ao mesmo tempo e com valores em ambos os casos próximos dos 50\%, e que já nos tinham suscitado dúvidas na sua inclusão num ou outro fator aquando da análise qualitativa (cf. Tabela 2). 


\section{KMO and Bartlett's Rest}

Kaiser-Meyer-Olkin Measure of Sampling Adequacy.

\begin{tabular}{ll}
.802 & \\
Approx. Chi-Square & 1243.441 \\
\hline df & 210 \\
\hline Sig. & .000
\end{tabular}

Bartlett's Test of Sphericity

Tabela 4 - Teste de KMO e Bartlett's com 21 itens

\begin{tabular}{|c|c|c|c|c|c|}
\hline item & 1 & 2 & 3 & 4 & 5 \\
\hline$V 1$ & 0.781 & & & & \\
\hline$V 2$ & 0.800 & & & & \\
\hline$V 3$ & & & & 0.580 & \\
\hline$V 4$ & & & & 0.610 & \\
\hline$V_{5}$ & & & & & 0.799 \\
\hline$V 6$ & 0.698 & & & & \\
\hline$V_{7}$ & 0.844 & & & & \\
\hline$V 8$ & 0.801 & & & & \\
\hline$V 9$ & 0.600 & & & & \\
\hline V1o & & & & & 0.560 \\
\hline$V_{11}$ & & 0.628 & & & \\
\hline$V 12$ & & 0.787 & & & \\
\hline$V 13$ & & -0.603 & & & \\
\hline$V 14$ & 0.494 & & & & \\
\hline$V 15$ & & 0.622 & & & \\
\hline$V 16$ & & & -0.658 & & \\
\hline$V 17$ & & & 0.69 & & \\
\hline$V 18$ & & & 0.628 & & \\
\hline$V 19$ & & & 0.526 & & \\
\hline $\mathrm{V} 2 \mathrm{O}$ & & & & & 0.451 \\
\hline$V 21$ & & & & 0.739 & \\
\hline \%Variância explicada & 20.063 & 16.893 & 7.713 & 5.943 & 5.076 \\
\hline
\end{tabular}

Tabela 5 - Análise fatorial com rotação varimax.

Após a eliminação de 11 itens decorrente das várias análises fatoriais realizadas, comparámos a inclusão dos 21 itens feita segundo a análise de conteúdo qualitativa reportada na tabela 
2 e a análise fatorial a 5 fatores que resolvemos reter. Existe uma coincidência quase item a item entre estes dois métodos de análise conforme se pode observar na tabela 6 , exceto para os itens 6, 11 e 28 que se organizaram num novo fator a que resolvemos chamar de "Motivação centrada no Ego" para a distinguir das outras 4 dimensões.

\begin{tabular}{|c|c|c|c|}
\hline Dimensões originais & Itens originais & Itens excluídos & Itens retidos \\
\hline 1. Motivação de Realização & $\begin{array}{l}V_{3}, V_{13}, V_{14}, V_{1} 6, V_{17}, V_{18}, V_{24} \\
V_{26}, V_{28}\end{array}$ & $V_{3}, V_{13}, V_{14}, V_{26}$ & $V_{16}, V_{17}, V_{18}, V_{24}$ \\
\hline 2. Recursos Internos & $\begin{array}{l}V 1, V 2, V 7, V 8, V 9, V 10, V_{15}, V_{21}, \\
V 22\end{array}$ & $V 15, V 21$ & $\begin{array}{l}V 1, V 2, V 7, V 8, V 9 \\
V 10, V 22\end{array}$ \\
\hline 3. Forças pessoais & $V_{12}, V_{19}, V_{25}, V_{27}, V_{3}, V_{31}, V_{32}$ & $V 12, V 19, V_{27}$ & $V_{25}, V_{30}, V_{31}, V_{32}$ \\
\hline 4. Hábitos de trabalho & $V_{4}, V 6, V_{11}, V_{20}, V_{23}, V_{29}, V_{5}$ & $V 2 O, V 23$ & $V_{4}, V_{5}, V_{29}$ \\
\hline \multirow[t]{2}{*}{$\begin{array}{l}\text { 5. Motivação centrada no } \\
\text { Ego }\end{array}$} & & & $V 6, V 11, V 28$ \\
\hline & 32 itens originais & 11 excluídos & 21 retidos \\
\hline
\end{tabular}

Tabela 6 - Relação dos itens entre Análise de Conteúdo e AFE

Após a AFE passámos a determinar a fiabilidade de cada um dos 5 fatores que emergiram e da escala na sua globalidade.

\subsection{Fiabilidade}

"A fiabilidade de uma medida refere a capacidade desta ser consistente. Se um instrumento de medida dá sempre os mesmos resultados (dados) quando aplicado a alvos estruturalmente iguais, podemos confiar no significado da medida e dizer que a medida é fiável.” (Maroco \& Garcia-Marques, p. 66)

A medida escolhida foi a da consistência interna determinada por meio do Alpha de Cronbach que, para Maroco e Garcia-Marques (2013), é definida como "uma medida estável de fiabilidade pois não está sujeita à variabilidade resultante da forma como o instrumento ou teste é dividido para calcular a fiabilidade split-half.” (p. 73).

Como a escala é multidimensional determinámos o valor de Alpha para cada um dos 5 fatores, conforme tabela 7 e para o conjunto dos 21 itens, conforme tabela 8.

O Coeficiente Alpha de Cronbach total foi de.723, que pode ser considerado como aceitável. Verificámos ainda que não havia qualquer vantagem em eliminar itens pois nenhum fazia subir o valor do alfa.

\begin{tabular}{llllll}
\hline & $\begin{array}{l}\text { Dimensão 1 } \\
\text { Motivação } \\
\text { realização }\end{array}$ & $\begin{array}{l}\text { Dimensão 2 } \\
\text { Recursos } \\
\text { internos }\end{array}$ & $\begin{array}{l}\text { Dimensão 3 } \\
\text { Forças Pessoais } \\
\text { internas }\end{array}$ & $\begin{array}{l}\text { Dimensão 4 } \\
\text { Hábitos de } \\
\text { trabalho }\end{array}$ & $\begin{array}{l}\text { Dimensão 5 } \\
\text { Motivação } \\
\text { centrada no Ego }\end{array}$ \\
\hline \multirow{2}{*}{ Itens } & $\begin{array}{l}V_{1}, V 2, V 7, V 8, \\
V 9, V 10, V 22\end{array}$ & $\begin{array}{l}V 16, V 17, V 18, \\
V 24\end{array}$ & $\begin{array}{l}\text { V25, V3o, V31, } \\
V 32\end{array}$ & V4, V5, V29 & V6, V11, V28 \\
\hline Alpha & 0.856 & -0.022 & 0.121 & 0.611 & 0.256 \\
\hline
\end{tabular}

Tabela 7 - Definição do Alpha para cada fator (5) 


\begin{tabular}{ll}
\hline Reliability Statistics \\
\hline Cronbach's Alpha & Nof items \\
\hline o.723 & 21 \\
\hline
\end{tabular}

Tabela 8 - Resultados obtidos do Coeficiente de Fiabilidade pelo Alpha de Cronbach Após a Análise da fiabilidade de cada fator e tendo obtido resultados inaceitáveis para os fatores 2, 3 e 5, resolvemos refazer a AFE com os itens que integravam os fatores 1 e 4, referentes à Motivação de Realização e às Hábitos de Trabalho. Apresentamos de seguida os resultados desta análise a 2 fatores com os 10 itens retidos, seguidos do Alpha por dimensão e total.

\section{KMO and Bartlett's Rest}

\begin{tabular}{lll}
\hline Kaiser-Meyer-Olkin Measure of Sampling Adequacy. & \multicolumn{2}{l}{} \\
\hline \multirow{3}{*}{ Bartlett's Test of Sphericity } & Approx. Chi-Square & 655.166 \\
\cline { 2 - 3 } & $\mathrm{df}$ & 45 \\
\cline { 2 - 3 } & Sig. & .000 \\
\hline
\end{tabular}

Tabela 9 - Teste de KMO e Bartlett's com 10 itens

\begin{tabular}{lll}
\hline item & 1 & 2 \\
\hline $1(V 1)$ & 0.774 & \\
\hline $2\left(V_{2}\right)$ & 0.799 & \\
\hline $3\left(V_{4}\right)$ & & 0.648 \\
\hline $4\left(V_{5}\right)$ & & \\
\hline $5(V 7)$ & 0.6900 \\
\hline $6(V 8)$ & 0.850 & \\
\hline $7(V 9)$ & 0.791 & \\
\hline $8(V 10)$ & 0.649 & \\
\hline $9\left(V_{22}\right)$ & 0.518 & 15.548 \\
\hline $10\left(V_{2} 8\right)$ & & \\
\hline \%Variância explicada & 39.678 & \\
\hline
\end{tabular}

Tabela 10 - Análise fatorial com rotação varimax com 2 dimensões.

\begin{tabular}{lll}
\hline & Reliability Statistics & \\
\hline Dimensão & Cronbach's Alpha & Nof items \\
\hline Dimensão 1 (Motivação de Realização) & 0.856 & 7 \\
\hline Dimensão 2 (Hábitos de Trabalho) & 0.611 & 3 \\
\hline
\end{tabular}

Tabela 11 - Resultados do Coeficiente de Fiabilidade pelo Alpha de Cronbach por Dimensão 


\begin{tabular}{ll}
\hline \multicolumn{2}{c}{ Reliability Statistics } \\
\hline Cronbach's Alpha & Nof items \\
\hline o.825 & 10 \\
\hline
\end{tabular}

Tabela 12 - Resultados do Coeficiente de Fiabilidade pelo Alpha de Cronbach (10 itens)

É esta a escala final que, para a nossa amostra, melhor representa a avaliação ou medida do constructo de Motivação de Realização (ver Apêndice A). Contudo, tratando-se de uma primeira análise fatorial do constructo será necessário realizar novas análises junto de outras amostras, usando a escala original com os 32 itens, para verificar como este instrumento se comporta.

\section{Conclusões}

Neste estudo foi apresentado o procedimento de validação de uma Escala para medir a Motivação de Realização de estudantes de cursos de Arquitetura para aprenderem a programação informática, que faz parte dos currículos da formação inicial dos arquitetos portugueses e brasileiros.

Os resultados apontam que a Escala escolhida, traduzida e validada para uma amostra de 204 estudantes portugueses e brasileiros, apresenta bons índices de sensibilidade dos itens, uma estrutura fatorial consistente com a teoria da motivação de realização, embora as quatro dimensões sugeridas pelos autores da escala não se tenham confirmado no nosso estudo, tendo sido retidas apenas duas dimensões que explicam cerca de $51 \%$ da variância total; uma dimensão relacionada diretamente com a Motivação de Realização (Fator ou Dimensão 1) e a outra com as Hábitos de Trabalho (Fator ou Dimensão 4). A consistência interna de cada fator e da escala no seu conjunto podem ser consideradas boas.

Deste modo podemos usar com segurança esta nova escala para medir a motivação de realização de estudantes de arquitetura portugueses e brasileiros que se estão a aprender a programação informática. No entanto, como em qualquer estudo de validação de um instrumento de medida, este precisa de ser validado em novas amostras de estudantes do ensino profissional e do ensino superior que tenham como língua oficial da escolarização o português, e que se estejam a iniciar na aprendizagem da atividade de programação. Seria também importante desenvolver estudos de validação com esta escala, traduzida e primeiramente validada para a amostra deste estudo, com estudantes cuja primeira opção é a aprendizagem da informática e com estudantes que frequentam outros cursos, mas onde existe uma disciplina obrigatória de programação. Estes estudos permitiriam dar maior credibilidade a este primeiro estudo de validação e garantir um maior poder de generalização dos resultados.

\section{Referências}

Almeida, L., \& Freire, T. (2017). Metodologia de investigação em Psicologia e Educação (5. ${ }^{\text {a }}$ Ed. Revista). Braga: Psiquilíbrios.

Anastasi, A., \& Urbina, S. (1997). Psychological testing (7th ed.). New Jersey: Library of Congress Cataloging-in-Publication Data. 
Atkinson, J. W. (1957). Motivational determinants of risk-taking behavior. Psychological Review, 64(6), 359-372.

Atkinson, J. W., \& Feather, N. T. (1969). A theory of achievement motivation. Australian Journal of Psychology, 21(3), 335-349.

Beirão, J. N. (2017). Sobre o ensino da Arquitectura e o futuro profissional do Arquitecto. O papel da Arquitectura nas sociedades criativas. Obtido em 20 de 04 de 2017, Jornal Arquitectos: http://www.jornalarquitectos.pt/pt/forum/cronicas/sobre-oensino-da-arquitetura-e-o-futuro-profissional-do-arquiteto

Caspersen, M. E., \& Kölling, M. (2009). STREAM: A first programming. Journal ACM Transactions on Computing Education, 9(1),1-29.

Celani, G. (2008). Teaching CAD programing to architecture students. Gestão \& Tecnologia de Projetos, 3(2), 1-23. https://doi.org/10.4237/gtp.v3i2.73

Chiavenato, I. (1999). Gestão de pessoas: o novo papel dos recursos humanos nas organizações. Rio de Janeiro: Campus.

Cohen, L., Manion, L., \& Morrison, K. (2006). Research methods in education (5th ed.). London: RoutledgeFalmer.

Covington, M. V. (1998). The will to learn: A guide for motivating young people. Cambridge: Cambridge University Press.

Dan, W., Cooper, S., \& Paush, R. (2000). Making the connection: programming with animating small world. In Proceedings of the Fifth Annual Conference on Innovation and Technology in Computer Science Education (pp. 41-44). New York: ACM. http://doi.org/10.1145/343048.343070

Dweck, C. S., \& Leggett, E. L. (1988). A social-cognitive approach to motivation and personality. Psychological Review, 95(2), 256-273. http://dx.doi. org/10.1037/0033-295X.95.2.256

Edwards, A. L. (1957). Techniques of attitude scale construction. New York, USA: Appleton-Century-Crofts.

Elliot, A. J., \& Dweck, C. S. (2005). Handbook of competence and motivation (Vol. 4). New York: The Guilford Press.

Fonseca, D., Pifarré, M., \& Redondo, E. (2013). Relación entre calidad percibida y afinidad emocional de imágenes arquitectónicas en función del dispositivo de visualización: Recomendaciones para su uso docente. RISTI - Revista Ibérica de Sistemas E Tecnologias de Informação, (11), 1-15. https://doi.org/10.4304/risti.11.01-15.

Jenkins, T. (2002). On the difficulty of learning to program. Proceedings of the Thirty Annual Conference of the LTSN Centre for Information and Computer Sciences (pp. 53-58). United Kingdom: Laugh Brought University.

Jesus, S. N. (2000). Motivação e formação de professores. Coimbra, Portugal: Quarteto.

Kaiser, H. F. (1958). The varimax criterion for analytic rotation in factor analysis. Psychometrika, 23, 187-200. 
Lemos, M. S. (2015). Motivação e aprendizagem. In G. L. Miranda \& S. Bahia. (2015). Psicologia da Educação: temas de desenvolvimento, aprendizagem e ensino (3a. ed.) (pp. 193-231). Lisboa, Portugal: Relógio D’Água Editores.

Lieury, A., \& Fenouillet, F. (1997). Motivação e sucesso escolar. (A. Patrão, Trad.). Lisboa: Editorial Presença (Obra original publicada em 1996).

Maroco, J. (2014). Análise Estatística com o SPSS Statistics (6. ${ }^{\mathrm{a}}$ ed.). Pêro Pinheiro, Portugal: Gráfica Manuel Barbosa \& Filho.

Maroco, J., \& Garcia-Marques, T. (2013). Qual a fiabilidade do alfa de Cronbach? Questões antigas e soluções modernas?. Laboratório de Psicologia, 4(1), 65-90.

McClelland, D. C., Atkinson, J. W., Clark, R. A., \& Lowell, E. L. (1953). The achievement motive. East Norwalk, Conn: Appleton-Century-Crofts.

Moons, J., \& Backer, C. (2013). The design and pilot evaluation of an interactive learning environment for introductory programming influenced by cognitive load theory and constructivism. Computers \& Education, 6o, 368-384. https://doi.org/10.1016/j. compedu.2012.08.009

Moreira, J. M. (2004). Questionários: Teoria e prática. Lisboa: Almedina.

Muthee, J. M., \& Thomas, I. (2011). Predictors of academic performance and motivation among Kenyan adolescents: a study among standard VIII pupils of Nairobi province. Department of Psychology. Retrieved from http://content.ebscohost. com.ezproxy.mtsu.edu/ContentServer. $\mathrm{T}=\mathrm{P} \& \mathrm{P}=\mathrm{AN} \& \mathrm{~K}=507984450$ \& $\mathrm{S}=\mathrm{R}$ $\& \mathrm{D}=$ eft $\&$ EbscoContent $=$ dGJyMNHX8kSeqa44v + vlOLCmroyeqLFSr6m4S6 + WxWXS\&ContentCustomer $=$ dGJyMPGqtE + wqq5OuePfgeyx43zx

Pea, R. D., \& Kurland, D. M. (1984). On the cognitive prerequisites of learning computer programming (Technical Report No 18). New York: Bank Street College of Education, Center for Children and Technology. https://doi.org/10.1016/0732$118 X(84) 90018-7$

Ramos, S. I. (2013). Motivação académica dos alunos do ensino superior. Obtido em 20 de 04 de 2017, de Psicologia.pt - O portal dos psicólogos: http://www.psicologia. pt/artigos/textos/A0677.pdf.

Runa, A. I. do N. F., \& Miranda, G. L. (2015). Validação Portuguesa das escalas de bemestar e mal-estar emocional. RISTI - Revista Ibérica de Sistemas e Tecnologias de Informação, 2015(16), 129-144. https://doi.org/10.17013/risti.16.129-144

Santos, D. M., \& Beirão, J. N. (2017). Generative tool to support architectural design decision of earthbag building domes. SIGraDi 2017- XXI Congreso de La Sociedad Ibero-Americana de Gráfica Digital, November (pp. 538-543). https://doi. org/10.5151/sigradi2017-083

Taipa, J. A., \& Fita, E. C. (2015). A motivação em sala de aula: o que é, como se faz?. São Paulo: Loyola.

Tuckman, B. W. (2012). Manual de investigação em educação (4. ${ }^{\mathrm{a}}$ ed.). Lisboa: Fundação Calouste Gulbenkian. 


\section{Apêndice A: Escala de Motivação de Realização}

\begin{tabular}{llllllll}
\hline No. & CA & QSA & PA & QSD & CD \\
\hline
\end{tabular}

1. Sinto que sou uma pessoa preguiçosa

2. Muitas vezes passam-se dias sem eu ter feito nada.

3. Planeio com antecedência quais os assuntos que vou

3. $\quad$ estudar durante o meu tempo livre.
Quando sei que alguém que gosta de mim conseguiu
4. alcançar algo grandioso, fico motivado para fazer a mesma coisa de uma maneira melhor.

5. Vou adiando o que eu deveria estudar no dia-a-dia.

6. Levo muito tempo para começar a estudar.
Na maioria dos dias prefiro descontrair e relaxar em
7. vez de me preparar para o próximo dia de estudo na Faculdade.

8. Às vezes esqueço-me de fazer as tarefas de casa.

Prefiro usar o meu tempo para fazer qualquer outra

9. coisa, ao contrário de tentar aperfeiçoar algo que já tenha concluído.

10. Gosto de terminar as minhas tarefas académicas,

10. mesmo quando são difíceis e consomem muito tempo. 